\title{
A case with Crohn's disease-associated spondyloarthritis exhibiting enhanced pro-inflammatory cytokine responses to Toll-like receptor ligands
}

\author{
Hajime Honjo, ${ }^{1}$ Tomohiro Watanabe, ${ }^{1}$ Natsuki Okai, ${ }^{1}$ Masashi Kono, ${ }^{1}$ Ken Kamata, ${ }^{1}$ Kosuke Minaga,${ }^{1}$ \\ Yoriaki Komeda, ${ }^{1}$ Shigeyoshi Tsuji, ${ }^{2}$ Masatoshi Kudo
}

\begin{abstract}
Background: Despite the high incidence of spondyloarthritis (SpA) as an extra-intestinal manifestation of Crohn's disease $(\mathrm{CD})$, the immunopathogenesis of CD-associated SpA remains largely unknown.

Objective: We tried to explore molecular mechanisms accounting for the development of CD-associated SpA in a patient successfully treated with infliximab.

Methods: Peripheral blood mononuclear cells (PBMCs) before infliximab treatment were stimulated with Toll-like receptor (TLR) ligands to measure pro-inflammatory cytokine responses. Endoscopic biopsy samples before and after infliximab treatment were subjected to quantitative polymerase chain reaction.

Results: PBMCs from this CD-associated SpA patient exhibited higher production of pro-inflammatory cytokines upon stimulation with TLR ligands than PBMCs from healthy controls. Induction of remission by infliximab was associated with the downregulation of pro-inflammatory cytokine responses in the small intestinal mucosa, which is continually exposed to TLR ligands.
\end{abstract}

Conclusion: Excessive pro-inflammatory cytokine responses to TLR ligands might underlie the immunopathogenesis of CD-associated SpA.

Key words: Crohn's disease, spondyloarthritis, Toll-like receptor, intestinal microflora, pro-inflammatory cytokines

\section{From:}

${ }^{1}$ Department of Gastroenterology and Hepatology,

Faculty of Medicine, Kindai University, Osaka-Sayama, Osaka, Japan

Department of Orthopedics/Rheumatology,

National Hospital Organization, Osaka Minami Medical Center,

Kawachi-Nagano, Osaka, Japan

\section{Introduction}

Spondyloarthritis (SpA) is among the most common extra-intestinal manifestations of Crohn's disease (CD). ${ }^{1}$ Despite the high incidence of CD-associated SpA, its immunopathogenesis is poorly understood. A common feature of CD and $\mathrm{SpA}$ immunopathogenesis is that pro-inflammatory cytokine responses, including IL-17, IL-23, and TNF- $\alpha$ mediate colitis and arthritis, as shown by recent therapeutic advances using biologics. ${ }^{1-3}$ In CD, such pro-inflammatory cytokine responses are triggered by intestinal dysbiosis. Thus, T helper type 1 and type 17 cells, that produce IFN- $\gamma$ and IL-17, respectively, accumulate in intestinal mucosa displaying dysbiosis.,

\section{Corresponding author:}

Tomohiro Watanabe

Department of Gastroenterology and Hepatology,

Kindai University Faculty of Medicine

377-2 Ohno-Higashi, Osaka-Sayama, Osaka 589-8511, Japan

E-mail: tomohiro@med.kindai.ac.jp

Interestingly, recent studies suggested that arthrogenic immune responses causing SpA might also originate from intestinal mucosa displaying dysbiosis. ${ }^{3}$ These data support the concept that the gut-joint immune axis creates an immune microenvironment for the development of SpA by promoting pro-inflammatory cytokine responses. Given such similarities between $\mathrm{CD}$ and SpA immunopathogenic processes, excessive pro-inflammatory cytokine responses to intestinal microflora likely mediate the development of CD-associated SpA. However, it remains unknown whether CD-associated $\mathrm{SpA}$ is characterized by excessive immune responses to 
intestinal microflora. Here, we report a case of CD-associated $\mathrm{SpA}$ in which excessive pro-inflammatory cytokine responses to Toll-like receptor (TLR) ligands derived from intestinal microflora might drive the development of both CD and SpA.

\section{Report of Case}

A 44-year-old woman was admitted to our hospital for further examination of high-grade fever $\left(>38.0^{\circ} \mathrm{C}\right)$, watery diarrhea, and abdominal pain. She also noticed finger stiffness, bilateral knee pain, and neck pain. Blood tests showed mild anemia, thrombocytosis (leukocyte count, $3.41 \times 10^{3} / \mu \mathrm{L}$; red blood cell count, $3.68 \times 10^{4} / \mu \mathrm{L}$; hemoglobin, $10.6 \mathrm{~g} / \mathrm{dL}$; hematocrit $34.1 \%$; platelet count, $\left.43.9 \times 10^{4} / \mu \mathrm{L}\right)$, and a marked elevation of C-reactive protein $(6.88 \mathrm{mg} / \mathrm{dL})$. Serum levels of $\mathrm{IgG}, \operatorname{IgA}$, and $\operatorname{IgM}$, as well as the serum anti-nuclear antibody titer, were within the normal range. Typing of human leukocyte antigen revealed B44 and B48.

Diffuse wall thickening of the jejunum and ileum was seen in abdominal computed tomography performed to investigate the cause of diarrhea. Double balloon-assisted enteroscopy (DBE) revealed multiple longitudinal ulcers and a cobblestone appearance in the jejunum and ileum (Figure 1a). Pathological examination of jejunum biopsy specimens detected an epithelioid granuloma and infiltration of lymphocytes and neutrophils (Figure 1b). Polymerase chain reaction (PCR) tests for detecting cytomegalovirus and tuberculosis were negative in the small intestinal biopsy specimens. Immunohistochemical analyses were performed to determine the type of immune cells that accumulated in the jejunal lesions, as described

(a)

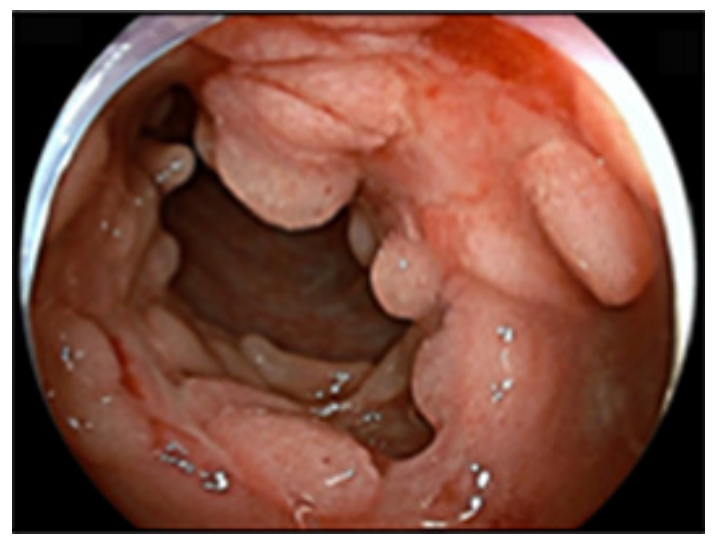

Before Infliximab previously. ${ }^{5} \mathrm{CD}^{+} \mathrm{T}$ cells and $\mathrm{CD} 20^{+} \mathrm{B}$ cells accumulated in the inflammatory lesions surrounding the epithelioid granuloma, whereas $\mathrm{CD}^{+} 8^{+}$macrophages accumulated in the epithelioid granuloma itself (Figure 1b). These endoscopic and pathological findings were fully consistent with the diagnosis of CD.

Radiological examinations were performed to investigate the cause of finger stiffness, bilateral knee pain, and neck pain. The presence of enthesitis of the hand was suspected on physical examination. Finger X-rays revealed cortical bone thickening and osteosclerosis in almost all of the distal and middle phalanges in the first, second, third, and fourth fingers bilaterally (Figure 1c). Cortical bone thickening and osteosclerosis were also observed in the proximal phalanges in the right fingers. Syndesmophytes were observed in her C6 and C7 vertebrae in the cervical X-ray (Figure 1d). Magnetic resonance imaging using short T1 inversion recovery showed a high intensity area in patient's right tibia, suggesting the presence of enthesitis of semitendinosus tendon (data not shown). These radiological findings supported the diagnosis of axial SpA. ${ }^{6}$ Thus, she was finally diagnosed with $\mathrm{CD}$-associated SpA. She was treated with infliximab (IFX, $5 \mathrm{mg} / \mathrm{kg}$ ) to induce remission of both active CD and SpA. DBE, which was performed four times after the injection of IFX, confirmed the healing of jejunal and ileal ulcers (Figure 1a). A marked reduction in immune cell infiltration was seen in pathological examinations using small intestinal biopsy specimens. Moreover, IFX treatment markedly improved both CD- and SpA-associated symptoms. Thus, IFX treatment successfully induced clinical remission of CD-associated $\mathrm{SpA}$.

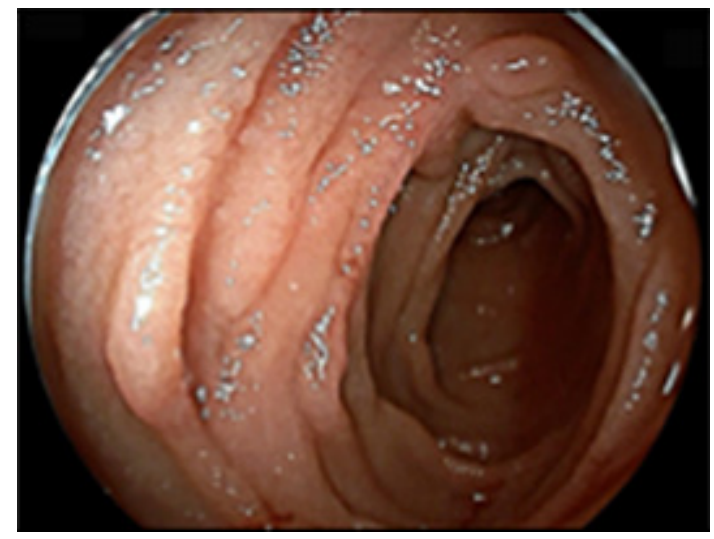

After Infliximab

Figure 1. A patient with Crohn's disease (CD)-associated spondyloarthritis (SpA) successfully treated with infliximab (IFX) treatment. (a) Endoscopic images of a patients with CD-associated SpA. Longitudinal ulcers and a cobblestone appearance are seen in the jejunal mucosa of this patient with CD-associated SpA before IFX treatment (left). Absence of active ulcers after IFX treatment (right). (b) Pathological findings in the jejunum in a patient with CD-associated SpA. Endoscopic biopsy samples obtained from the jejunum before IFX treatment were fixed in $10 \%$ formalin followed by hematoxylin and eosin (H\&E) staining. This revealed epithelioid granuloma and massive infiltration of lymphocytes and neutrophils. Biopsy samples were also subjected to immunohistochemical analyses using anti-CD3, anti-CD20, and anti-CD68 antibodies to determine the type of immune cells that were present. Scale bar: $250 \mu \mathrm{m}$. (c, d) Radiological images in a patient with CD-associated SpA. Cortical bone thickening and osteosclerosis is evident in almost all distal and middle phalanges in the first, second, third, and fourth fingers bilaterally (c, white arrows). Cortical bone thickening and osteosclerosis were also observed in the proximal phalanges in the right fingers (c, white arrows). Syndesmophytes were observed in her C6 and C7 vertebrae in the cervical X-ray (d, yellow arrows). 
(b)

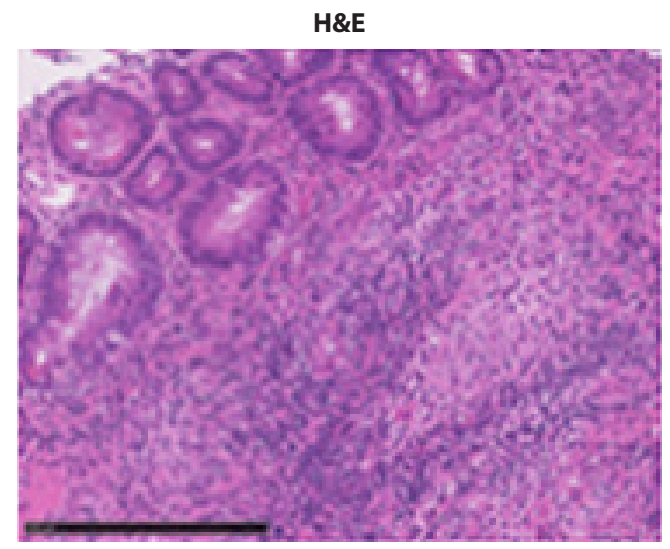

CD20

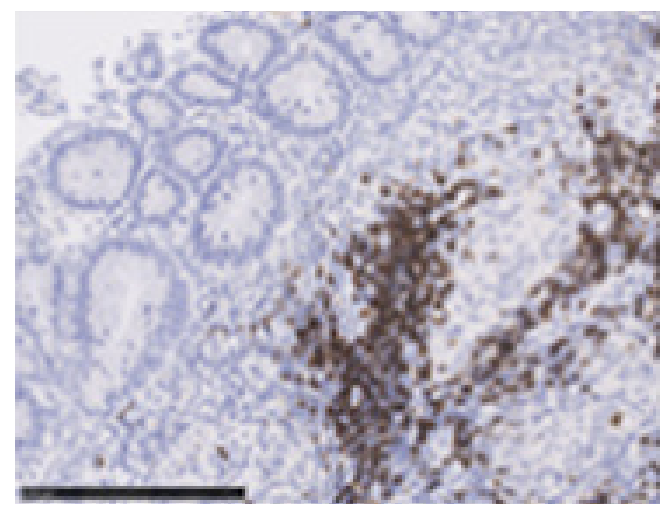

(c)

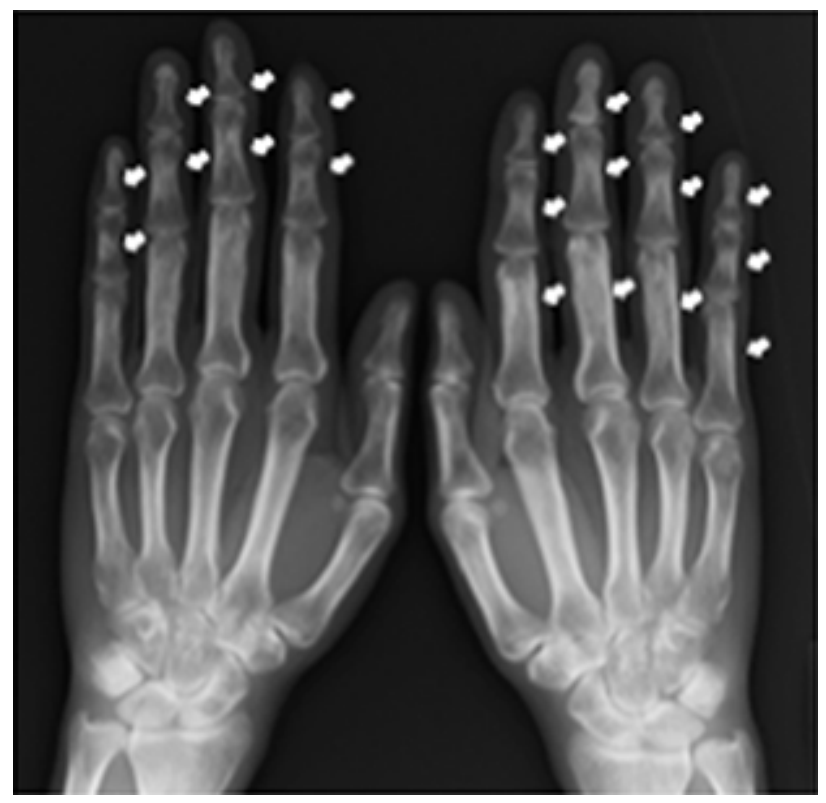

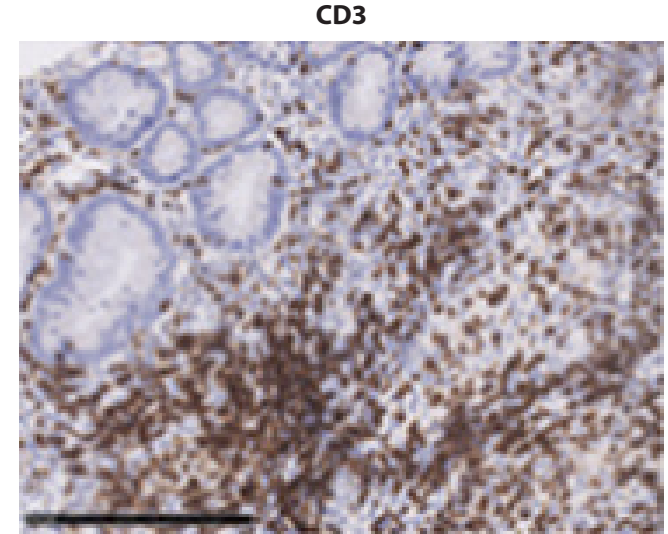

CD68

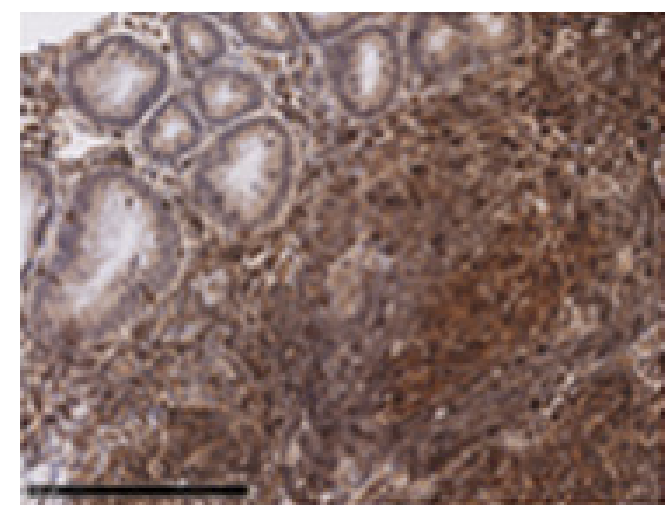

(d)

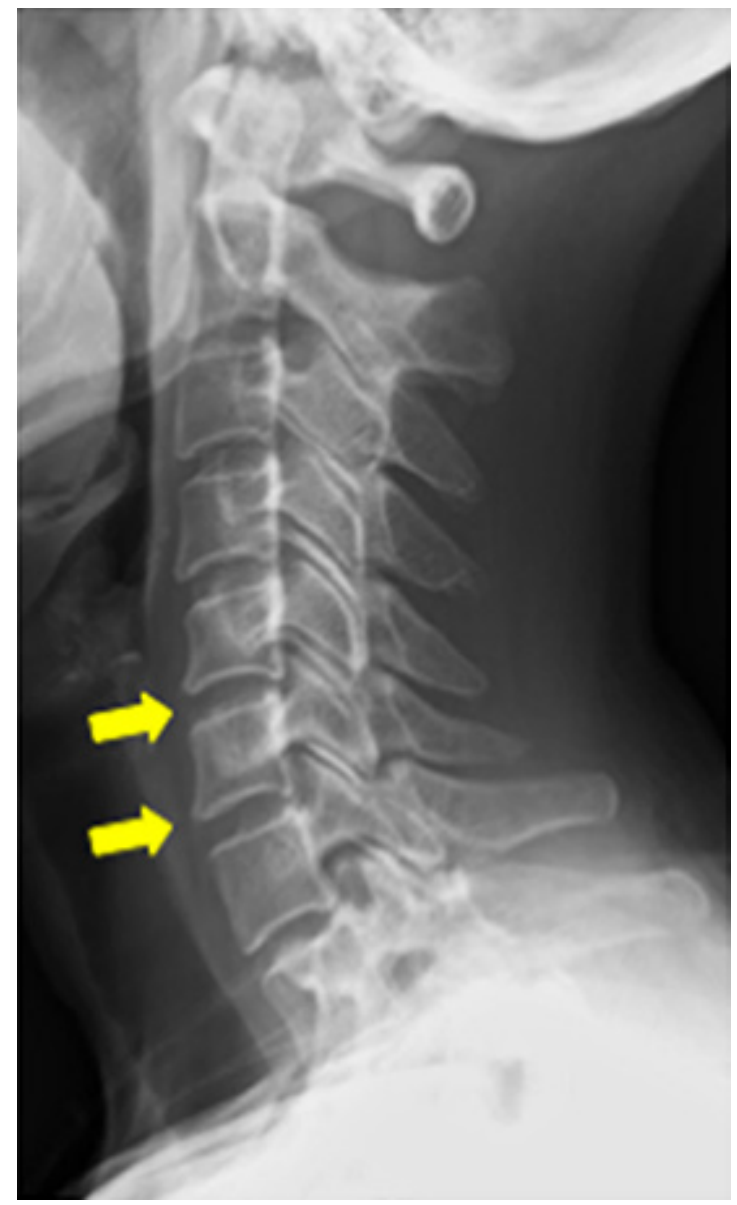


(a)

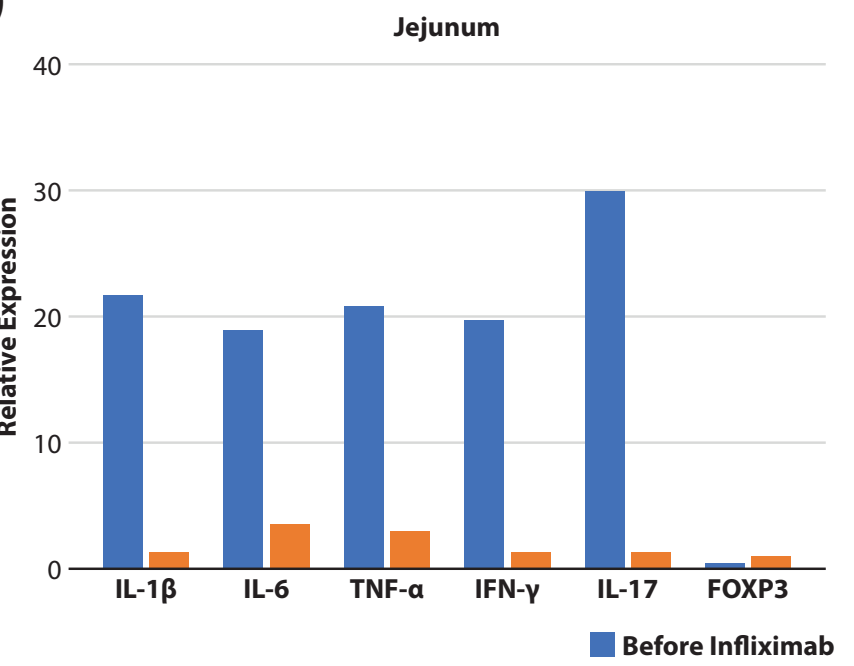

(b)
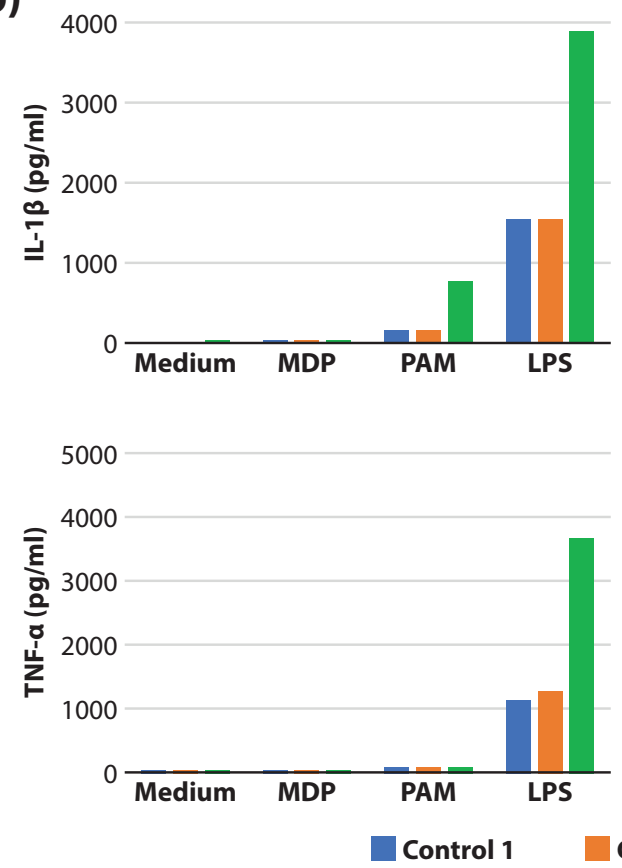

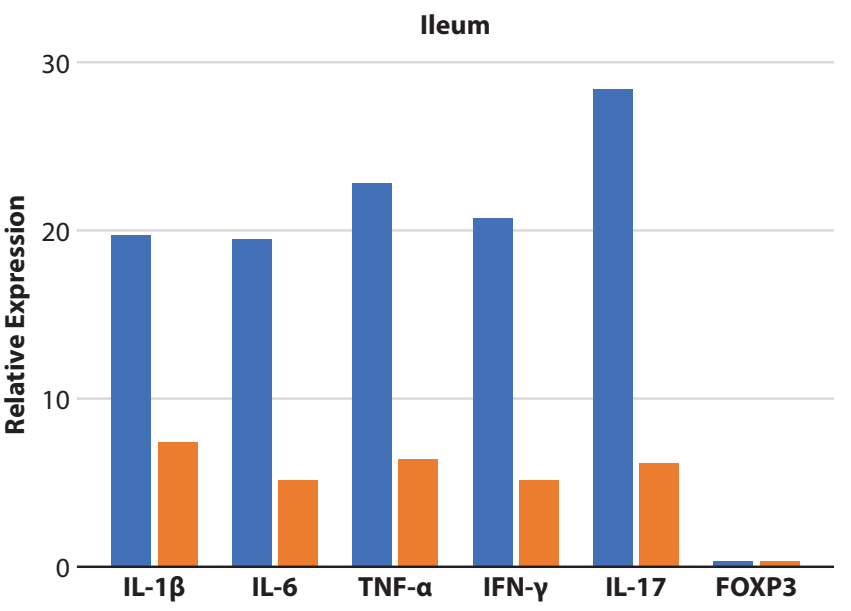

After Infliximab

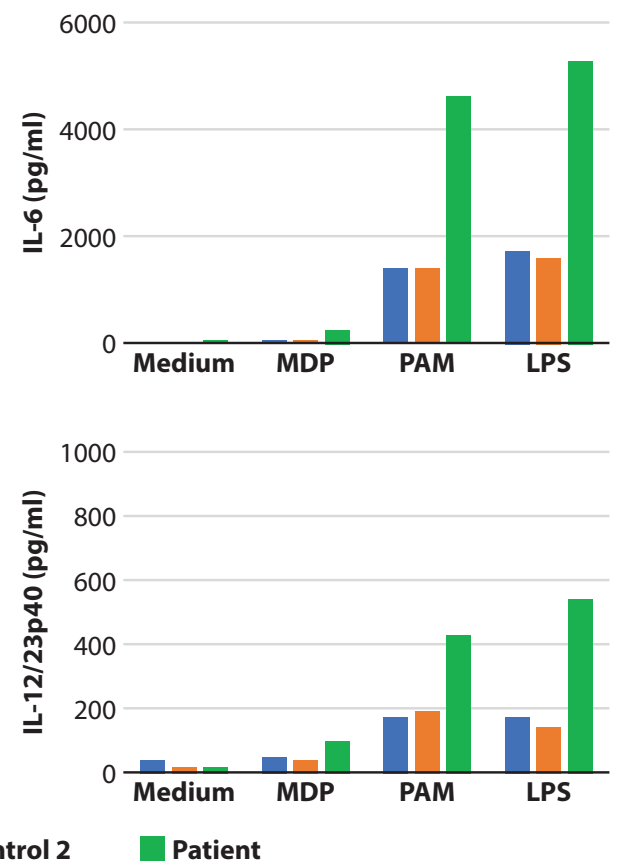

Figure 2. Excessive production of pro-inflammatory cytokines in response to Toll-like receptor ligands in a patient with Crohn's disease (CD)-associated spondyloarthritis (SpA). (a) Cytokine profiles of the jejunum and ileum of the patient before and after infliximab (IFX) treatment. Total mRNA was isolated from the biopsy samples and subjected to the quantitative reverse transcription polymerase chain reaction to determine the expression levels of IL-1 $\beta$, IL-6, TNF- $\alpha$, IFN- $\gamma$, IL-17, and forkhead box p3 (FOXP3) mRNAs. Expression levels were normalized to that of $\beta$-actin. (b) Peripheral blood mononuclear cells $\left(2 \times 10^{6} / \mathrm{mL}\right)$ isolated from the patient and two healthy controls (Control 1, Control 2) were treated with muramyl dipeptide (MDP, a NOD2 ligand, $10 \mu \mathrm{g} / \mathrm{mL}$ ), PAM ${ }_{3}$ CSK4 (PAM, a TLR2 ligand, $10 \mu \mathrm{g} / \mathrm{mL}$ ), or lipopolysaccharide (LPS, a TLR4 ligand, $1 \mu \mathrm{g} / \mathrm{mL}$ ) for $48 \mathrm{~h}$. Then, the levels of pro-inflammatory cytokines (IL-1 $\beta$, IL-6, TNF- $\alpha$, IL-12/23p40) were determined by enzyme-linked immunosorbent assays.

To determine the molecular mechanisms underlying IFXinduced clinical remission in CD and SpA, mRNA was isolated from the jejunal and ileal biopsy specimens before and after IFX treatment, and subjected to quantitative PCR analysis as described previously. ${ }^{7}$ Because excessive innate immune responses to intestinal microbiota underlie the immunopathogenesis of not only $\mathrm{CD}$, but also $\mathrm{SpA},{ }^{1-3}$ peripheral blood mononuclear cells (PBMCs) isolated from this patient and two healthy controls were stimulated with nucleotidebinding oligomerization domain 2 (NOD2) and TLR ligands to measure the levels of pro-inflammatory cytokines (IL- $1 \beta$, IL-6, TNF- $\alpha$ and IL-12/23p40) as described previously. ${ }^{8}$ Briefly, PBMCs were stimulated with the NOD2 ligand, muramyl dipeptide $(10 \mu \mathrm{g} / \mathrm{mL})$, the TLR2 ligand, PAM ${ }_{3}$ CSK4 $(10 \mu \mathrm{g} /$ $\mathrm{mL})$, and the TLR4 ligand, lipopolysaccharide $(1 \mu \mathrm{g} / \mathrm{mL})$. Ethical permission for this study was obtained from the Review 
Board of the Kindai University Faculty of Medicine. Written informed consent was obtained from the patient and healthy controls.

Induction of remission by IFX markedly reduced expression of the pro-inflammatory cytokines IL-1 $\beta$, IL-6, TNF- $\alpha$, IFN- $\gamma$, and IL-17 in both the jejunal and ileal mucosa (Figure 2a). Such suppressive effects on broad ranges of cytokine responses by IFX can be partially explained by the fact that expression of pro-inflammatory cytokines requires activation of nuclear factor-kappa B, a prototypical transcription factor activated by TNF- $a{ }^{4}$ In contrast, expression of forkhead box P3, a master regulator of regulatory $\mathrm{T}$ cells, was comparable before and after IFX treatment in both the jejunal and ileal mucosa. Thus, the induction of remission by IFX was accompanied by a reduction of pro-inflammatory responses rather than an accumulation of regulatory $\mathrm{T}$ cells.

We next examined whether PBMCs isolated from this case before IFX treatment exhibited excessive innate immune responses to NOD2 and TLR ligands derived from intestinal microbiota. We found that PBMCs from this patient produced large amounts of IL- $1 \beta$, IL- 6 , TNF- $\alpha$, and IL-12/23p40 upon stimulation with the NOD2 and TLR ligands as compared with PBMCs from healthy controls (Figure 2b), suggesting that excessive pro-inflammatory cytokine responses against intestinal microflora could underlie immunopathogenesis in this case.

\section{Discussion}

Although SpA signs are common extra-intestinal manifestations of active $\mathrm{CD},{ }^{1}$ the molecular mechanisms accounting for the link between CD and SpA are poorly defined. We encountered a case with concurrent occurrence of small intestinal CD and SpA, and analyzed pro-inflammatory cytokine responses. We found that PBMCs isolated from our patient exhibited enhanced production of pro-inflammatory cytokines upon stimulation with the NOD2 and TLR ligands, as compared with PBMCs from healthy controls. Moreover, quantitative PCR analyses of ileal and jejunal biopsy samples revealed a marked reduction of pro-inflammatory cytokine levels after successful induction of remission by IFX. Because NOD2 and TLR ligands are small molecules derived from intestinal microflora, these data suggest that excessive pro-inflammatory cytokine responses against intestinal microflora might underlie the immunopathogenesis of CD-associated SpA. In addition, this case report lends strong support to the hypothesis that pathogenic immune responses causing both $\mathrm{CD}$ and SpA might originate from gut mucosa, which is exposed continually to intestinal microbiota. ${ }^{1-3}$ However, we have to be cautious regarding the interpretation of these data since we have not analyzed pro-inflammatory cytokine responses in the joints. Moreover, it remains unknown whether excessive pro-inflammatory cytokine responses seen in the PBMCs could be reproduced in the intestine and joint. Differences in the percentages of peripheral blood monocytes or dendritic cells and in the expression of TLRs might affect the production of pro-inflammatory cytokines by PBMCs. More importantly, analyses of cytokine responses in a large number of patients with $\mathrm{CD}$-associated and CD-non-associated $\mathrm{SpA}$ are necessary to verify the link between the gut-joint axis and CD-associated SpA. Therefore, confirmation of this idea awaits future studies measuring pro-inflammatory cytokine responses in the intestine, joint, and peripheral blood in patients with SpA.

As for the pathogenic cytokine responses shared by $C D$ and $\mathrm{SpA}$, recent studies have highlighted the importance of IL-17, IL-23, and TNF- $a$. $^{1-4,}$ 9-11 Consistent with those findings, induction of remission by IFX markedly decreased the expression of IL-17 and TNF- $\alpha$ in the ileal and jejunal mucosa in this case. Moreover, PBMCs from a patient with CD-associated SpA produced a large amount of IL-12/23p40 upon stimulation with microbial antigens. Therefore, it is logical to assume that CD-associated SpA could be successfully treated by decreasing IL-17 or TNF-a levels. However, blocking of IL-17, which is necessary for maintenance of intestinal barrier integrity, ${ }^{12}$ did not induce remission in active CD patients. ${ }^{13}$ Moreover, IL-17 inhibition can result in intestinal inflammation in a significant fraction of SpA patients because of impairments in barrier function and gut microbiome alterations. ${ }^{14}$ Considering these pro-inflammatory, rather than anti-inflammatory, effects of neutralizing IL-17, inhibition of TNF- $\alpha$-mediated signaling pathways may be preferred for treating active CD-associated SpA. Indeed, IFX treatment successfully induced remission in both $\mathrm{CD}$ and $\mathrm{SpA}$ in the present case.

In conclusion, we report a case with CD-associated $\mathrm{SpA}$ exhibiting enhanced production of pro-inflammatory cytokines to TLR ligands. This case report suggests that excessive pro-inflammatory cytokine responses to TLR ligands might underlie the immunopathogenesis of CD-associated SpA. Confirmation of this idea awaits future studies addressing pro-inflammatory cytokine responses to TLR ligands in a large number of patients with $\mathrm{CD}$-associated $\mathrm{SpA}$ and CD-non-associated SpA.

\section{Acknowledgements}

This work was supported by a Grant-in-Aid for Scientific Research (19K08455) from the Japan Society for the Promotion of Science, and grants from the Takeda Science Foundation, Smoking Research Foundation, Yakult Bio-Science Foundation, and SENSHIN Medical Research Foundation. Hajime Honjo, Tomohiro Watanabe, Natsuki Okai, Masashi Kono, Ken Kamata, Kosuke Minaga, Yoriaki Komeda, Shigeyoshi Tsuji, and Masatoshi Kudo declare that they have no conflicts of interest. Hajime Honjo and Tomohiro Watanabe performed experiments and wrote the manuscript draft. Hajime Honjo, Natsuki Okai, Masashi Kono, and Yoriaki Komeda took care of the patient. Ken Kamata, Kosuke Minaga, and Masatoshi Kudo revised the manuscript.

\section{References}

1. Kumar A, Lukin D, Battat R, Schwartzman M, Mandl LA, Scherl E, et al. Defining the phenotype, pathogenesis and treatment of Crohn's disease associated spondyloarthritis. J Gastroenterol. 2020;55(7):667-78.

2. Caruso R, Lo BC, Nunez G. Host-microbiota interactions in inflammatory bowel disease. Nat Rev Immunol. 2020;20(7):411-26.

3. Simone D, Al Mossawi MH, Bowness P. Progress in our understanding of the pathogenesis of ankylosing spondylitis. Rheumatology (Oxford). 2018;57(suppl_6):vi4-vi9.

4. Strober W, Fuss IJ. Proinflammatory cytokines in the pathogenesis of inflammatory bowel diseases. Gastroenterology. 2011;140(6):1756-67. 
APJAI

5. Kono M, Komeda Y, Sakurai T, Okamoto A, Minaga K, Kamata K, et al. Induction of Complete Remission by Azacitidine in a Patient with Myelodysplastic Syndrome-Associated Inflammatory Bowel Disease. J Crohns Colitis. 2018;12(4):499-502.

6. Rudwaleit M. New approaches to diagnosis and classification of axial and peripheral spondyloarthritis. Curr Opin Rheumatol. 2010;22(4):375-80.

7. Watanabe T, Minaga K, Kamata K, Sakurai T, Komeda Y, Nagai T, et al. RICK/RIP2 is a NOD2-independent nodal point of gut inflammation. Int Immunol. 2019;31(10):669-83.

8. Watanabe T, Asano N, Murray PJ, Ozato K, Tailor P, Fuss IJ, et al. Muramyl dipeptide activation of nucleotide-binding oligomerization domain 2 protects mice from experimental colitis. J Clin Invest. 2008;118(2):545-59.

9. Ciccia F, Bombardieri M, Principato A, Giardina A, Tripodo C, Porcasi R, et al. Overexpression of interleukin-23, but not interleukin-17, as an immunologic signature of subclinical intestinal inflammation in ankylosing spondylitis. Arthritis Rheum. 2009;60(4):955-65.

10. Kaaij MH, van Tok MN, Blijdorp IC, Ambarus CA, Stock M, Pots D, et al. Transmembrane TNF drives osteoproliferative joint inflammation reminiscent of human spondyloarthritis. J Exp Med. 2020;217(10).
11. Viladomiu M, Kivolowitz C, Abdulhamid A, Dogan B, Victorio D, Castellanos JG, et al. IgA-coated E. coli enriched in Crohn's disease spondyloarthritis promote TH17-dependent inflammation. Sci Transl Med. 2017;9(376).

12. Lee JS, Tato CM, Joyce-Shaikh B, Gulen MF, Cayatte C, Chen Y, et al Interleukin-23-Independent IL-17 Production Regulates Intestinal Epithelial Permeability. Immunity. 2015;43(4):727-38.

13. Hueber W, Sands BE, Lewitzky S, Vandemeulebroecke M, Reinisch W, Higgins PD, et al. Secukinumab, a human anti-IL-17A monoclonal antibody, for moderate to severe Crohn's disease: unexpected results of a randomised, double-blind placebo-controlled trial. Gut. 2012;61(12): 1693-700.

14. Manasson J, Wallach DS, Guggino G, Stapylton M, Badri MH, Solomon G, et al. Interleukin-17 Inhibition in Spondyloarthritis Is Associated With Subclinical Gut Microbiome Perturbations and a Distinctive Interleukin-25-Driven Intestinal Inflammation. Arthritis Rheumatol. 2020;72(4):645-57. 